Mini Review

\title{
Convergence and the imaginary number
}

\begin{abstract}
The aim of this paper is to show that mathematics converges on one solution. At the heart of the issue is determining a value for " $i$ " the imaginary number. We provide here a brief explanation of the derivation of the imaginary number. Our results relate $0,1, \pi$, base $e$ , $\sqrt{2}, \sqrt{3}, i$, and the golden mean 1.618 -the key parameters of Applied Mathematics. We see that mathematics converges upon one solution which manifested in the physical universe.
\end{abstract}

Keywords: imaginary number, fibonacci sequence, astro-theology math, physics, mathematical constant

\author{
Volume I Issue 2 - 2018
}

\section{Paul TE Cusack}

Park Ave, Saint John, Canada

Correspondence: Paul TE Cusack, Dule 23 Park Ave, Saint John, NB E2J IR2, Canada, Email st-michael@hotmail.com

Received: February 27, 2018 | Published: April 17, 2018

\section{Introduction}

Everything in the universe is in motion. A static point is a special case that really doesn't exist. It is just a frame of reference convenient for analysis. The laws of Physics work for an observer in motion; or also for a point in motion observing the same thing. As Einstein had it, the laws are all relative depending upon the observer.

We have two points A and B separated by a distance of $1 \mathrm{~km}$, if we move from point A toward point B by half the distance, we still have $1 / 2$ a Km to go. Take half again; we have of course $1 / 4$ of a $\mathrm{km}$. If we keep taking half the distance, how do we ever get to point B?

The answer lies in that as we move toward point $\mathrm{A}$, point $\mathrm{A}$ moves toward us. So half the distance from $A$ to $B=1 / 2$ where they meet. If the distance is $\mathrm{x}$, instead of half, we get the following series:

$$
\begin{aligned}
& f(x)=1 /[1+x] \\
& f(f(x))=1 /[1+[1 /(1+x)] \\
&= \text { etc.... } \\
&= 0.618034=\mathrm{i} \\
& i=9.8066 / 0.15585=g /[1-\sin 1]=0.618=g / \text { Moment }
\end{aligned}
$$

The numerator is a Fibonacci sequence. Where they converge is at the imaginary number, "I" which is the conjugate of the Golden Mean. " $i$ " is the fraction of a decimal. It is where infinite mathematics meets finite mathematics. Or, $y=y^{\prime}=e^{x}$ Note that the Mathematical Constant, base $e$, can be expressed as a nested fraction also. So can Pi. Take the derivative of that function

\section{Result}

$1 /(x-1)^{2}$ Centres around " 1 "; the essence of mathematics. Everything is defined in terms of one. $\operatorname{Ln}(0 t)=\operatorname{Ln}(0)=1$ Mathematics is the study of the number " 1 " and its relations (which is defined as something as opposed to nothing) and the tension created by the constants. One salt shaker contains thousands of grains of salt. One grain of salt contains millions of atoms of $\mathrm{NaCl}$. "One" is a definition: a mental construct. ${ }^{1,2}$

When $t=0$, we are outside the physical universe. Mathematics is the only thing that survives out the universe where there is no time.
Refer to AT math for more on the physics this creates (Figure 1).

Since the fraction converges at 15 decimals

$$
\begin{aligned}
& 1 /(15-1)^{2} \\
& =1 / 14^{2}
\end{aligned}
$$

$1 / 196,196$ is a convergent number. $1 / 196 \sim=0=1 /$ infinity

Now for some AT Math

$\sqrt{3}-\sqrt{2}=1 / \pi$

$1 / \pi=$ frequency of the human mind

But, we know from AT Math

$\sqrt{3}=$ time $=\mathrm{t}=$ eigenvector

and

$\sqrt{2}=1 / v=t / d$

But $v=d / t$

so, $\sqrt{2}=1 / v=t / d$

so,

$1 / \pi=1 /(\sqrt{3}-\sqrt{2})$

$=1 /(t-t / d)$

$=1 /(t d-t) / d$

$=d /(t d-t)$

Let $t=1$

$=d /(d-1)$

$=x /(x-1)$

$=1 / \pi=1 / t=E$

So, $1 / t=x /(x-1)$

$(x-1)(1)=t x$

$-t x-x+1=0$

Let $\mathrm{t}=\mathrm{x}$ 
$x^{2}-x+1=0$ Golden Mean Parabola and we know

$\sqrt{3}+\sqrt{2}=\pi$

This simply changes the minus sign to a positive

$x+1-x=0$

$x+1-x=0$

$x=t=1 ; 0$

Discrete meets the continuous. $\operatorname{Ln}(0)=1$

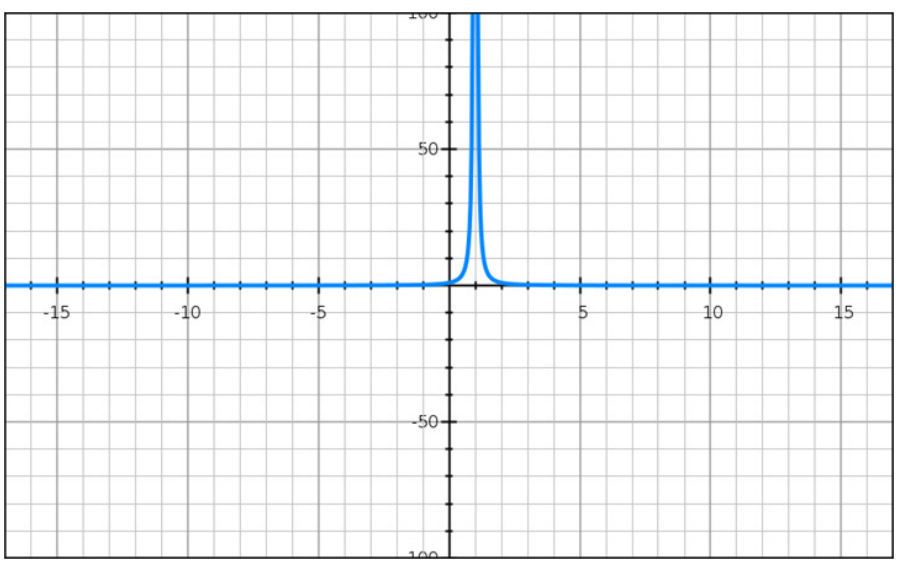

Figure 1 Plot of the derivative $1 /(x-1)^{2}$.

\section{Conclusion}

When you have motion, you necessarily have velocity, of $d=v / t$. Therefore, you have time. Time is K.E.; everything inside the universe is subject to time and motion. Outside the universe, the static condition is timeless or eternal, or also named God. God is outside of time. He is eternal. The best way to express God is through mathematics. There is a limit to amount of math ones need to know up till we see convergence. There is only one solution sand it's really the only one we need to know. The frequency of the human mind, the golden mean, and irrational roots all converge in this solution. This convergence of mathematics is important in helping us understand how and why the universe came to be. There was no choice.

\section{Acknowledgement}

None.

\section{Conflict of interest}

The author declares that there is no conflict of interest.

\section{References}

1. Paul TE Cusack. Convergence of Physical Mathematics and the Universal Torus. AJMS. 2017;1(3).

2. Eugene Don. Schaum's Outline of Mathematica 2nd ed. USA: McGraw Hill; 2009. p. 384. 